Letters to the Editor

\section{Adherence to iron supplementation in pregnancy}

\section{Dear editor:}

We read with great interest the valuable article, "Sociodemographic factors influencing adherence to antenatal iron supplementation recommendations among pregnant women in Malawi: Analysis of data from the 2010 Malawi Demographic and Health Survey". In this paper, the authors investigated the relationship between sociodemographic factors and adherence to iron supplementation in pregnant women. To do so, they analysed secondary data from the 2010 Malawi Demographic and Health Survery (DHS), which used self-reporting to assess adherence. Inaccurate patient recall is a major limitation of these data, and we believe that the findings should be interpreted with caution in this context (patient adherence may be overestimated). There are several direct and indirect methods to assess adherence to medications, each method has advantages and limits. Many studies have shown that pill counting is more accurate than self-reported adherence. ${ }^{2}$

On the other hand, patient haemoglobin levels were not reported. In a study conducted in Ethiopia, Bekele Taye and colleagues reported a significant association between anaemia history during the previous pregnancy and adherence to iron supplementation. ${ }^{3}$ Hence, in studies assessing adherence to iron supplementation, haemoglobin level testing would be a useful tool.

Finally, maternal and foetal morbidity related to iron deficiency during pregnancy may affect adherence to therapy. This was demonstrated by Samson Gebremedhin et al., ${ }^{4}$ and this finding is supported by the results of our own study on medication adherence among hypertension patients. Educational programmes should focus effectively on the health benefits of iron supplementation during pregnancy, especially in developing countries.

\section{Mohammed El Amine Ghembaza}

Department of Internal Medicine, Dr Tidjani Damerdji Hospital of

Tlemcen, Tlemcen, Algeria

amineghembaza@gmail.com

\section{Ali Louinici}

Department of Internal Medicine, Dr Tidjani Damerdji Hospital of Tlemcen, Tlemcen, Algeria

\section{References}

1. Titilayo A, Palamuleni ME, Omisakin O. Sociodemographic factors influencing adherence to antenatal iron supplementation recommendations among pregnant women in Malawi: Analysis of data from the 2010 Malawi Demographic and Health Survey. Malawi Med J. 2016 : Mar;28(1):1-5.

2. Ibrahim ZM, El-hamid SA, Mikhail $\mathrm{H}$ et al. Assessment of adherence to iron and folic acid Ssupplementation and prevalence of anemia in pregnant women. Med J Cairo Univ 2011 : 79(2):115-121.

3. Taye B, Abeje G, Mekonen A. Factors associated with compliance of prenatal iron folate supplementation among women in Mecha district, Western Amhara: a cross-sectional study. Pan Afr Med J , 2015 : vol. 20, article 43 .

4. Gebremedhin S, Samuel A, Mamo G et al. Coverage, compliance and factors associated with utilization of iron supplementation during pregnancy in eight rural districts of Ethiopia: a cross-sectional study. BMC Public Health. 2014 :vol. 14,no. 1, article 607.

5. Ghembaza MA, Senoussaoui Y, Tani MK et al. Impact of patient knowledge of hypertension complications on adherence to antihypertensive therapy. Curr Hypertens Rev. 2014 :10(1):41-8.

\section{Competing interests}

The authors declare that they have no conflicts of interest.

\section{Authors' reply:}

We would like to restate that we employed one of the 4 indirect ways (self-reporting-others being electronic monitoring, pill counts, and pharmacy refill rates) widely used in literature to measure adherence to medication, ${ }^{2}$ and that none of these widely used methods is devoid of its own disadvantages.

The 2010 Malawi Demographic and Health Survey (MDHS) was a cross-sectional survey, therefore establishing causality among variables must be considered with caution. Note that this generally applies to all cross-sectional surveys.

There was no way our analysis on adherence could have been through pill counting due to retrospective nature of reporting engaged in the survey

One hundred percent accuracy or certainty could not be guaranteed due to recall bias. However we tried minimising the effect of recall bias by limiting the birth history to the last 3 years before the survey (this was stated under the Methods section)

Though the 2010 MDHS contains haemoglobin levels of the respondents, we were not particularly focusing on anaemic participants in our study; we were more concerned with the rate of adherence to the routine of iron supplementation among all pregnant women regardless of haemoglobin level. We therefore recommended early antenatal care attendance (during the first trimester) so that women can receive advice on routine iron supplementation early in pregnancy and benefit from the entirety of the antenatal care package (this was stated in the Conclusions section). Take, for instance, the variation in prevalence of iron deficiency anaemia among women in rural and urban Malawi. Despite the higher prevalence in rural Malawi, as reported by Van den Broek et al., ${ }^{3}$ respondents who were urban residents still exhibited greater adherence to iron supplementation than their rural counterparts.

Despite all of these points, our findings on adherence were comparable to other reports in literature. ${ }^{3,4,5}$

\section{Ayotunde Titilayo}

Department of Demography \& Social Statistics, Obafemi Awolowo University, Ile-Ife, Nigeria liasuayotunde@gmail.com

\section{Martin E. Palamuleni}

Population Training \& Research Unit, North-West University, Mafikeng, South Africa

\section{Olusola Omisakin}

Department of Demography \& Social Statistics, Obafemi Awolowo University, Ile-Ife, Nigeria

\section{References}

1. Titilayo A, Palamuleni ME, Omisakin O. Socio-demographic factors influencing adherence to antenatal iron supplementation recommendations among pregnant women in Malawi: Analysis of data from the 2010 Malawi Demographic and Health Survey. Malawi Med J. 2016: Mar;28(1):1-5.

2. Hawkshead J, Krousel-Wood MA. Techniques for measuring medication adherence in hypertensive patients in outpatient settings: Advantages and limitations. Disease Management \& Health Outcomes. 2007; 15(2): 109118.

3. Van den Broek NR, Rogerson SJ, Mhango CG, Kambala B, White SA, Molyneux ME. Anaemia in pregnancy in southern Malawi: prevalence and risk factors. BJOG. 200 Apr;107(4):445-51.

4. Taye B, Abeje G, Mekonen A. Factors associated with compliance of prenatal iron folate supplementation among women in Mecha district, Western Amhara: a cross-sectional study. Pan Afr Med J. 2015 Jan 15;20:43. Doi:10.11604/pamj.2015.20.43.4894.

5. Titilayo A, Agunbiade OM. Iron deficiency anemia in pregnancy: implication for reproductive health in Nigeria. Journal of Demography and Social Statistics. 2014;1(1):98-109.

\section{Competing interests}

The authors declare that they have no conflicts of interest. 